Marquette University

e-Publications@Marquette

Biomedical Engineering Faculty Research and

Publications

Biomedical Engineering, Department of

8-2012

\title{
Upper Extremity Biomechanical Model for Evaluation of Pediatric Joint Demands during Wheelchair Mobility
}

\author{
Alyssa J. Paul \\ Marquette University \\ Brooke A. Slavens \\ Marquette University, brooke.slavens@marquette.edu \\ Adam Graf \\ Shriners Hospitals for Children \\ Joseph Krzak \\ Shriners Hospitals for Children \\ Lawrence Vogel \\ Shriners Hospitals for Children
}

See next page for additional authors

Follow this and additional works at: https://epublications.marquette.edu/bioengin_fac

Part of the Biomedical Engineering and Bioengineering Commons

\section{Recommended Citation}

Paul, Alyssa J.; Slavens, Brooke A.; Graf, Adam; Krzak, Joseph; Vogel, Lawrence; and Harris, Gerald F., "Upper Extremity Biomechanical Model for Evaluation of Pediatric Joint Demands during Wheelchair Mobility" (2012). Biomedical Engineering Faculty Research and Publications. 41.

https://epublications.marquette.edu/bioengin_fac/41 


\section{Authors}

Alyssa J. Paul, Brooke A. Slavens, Adam Graf, Joseph Krzak, Lawrence Vogel, and Gerald F. Harris 


\title{
Upper Extremity Biomechanical Model For Evaluation Of Pediatric J oint Demands During Wheelchair Mobility
}

\author{
Brooke A. Slavens \\ Department of Occupational Science and Technology at the University of Wisconsin- \\ Milwaukee (UWM), \\ Milwaukee, $W I$ \\ Adam Graf \\ Shriners Hospitals for Children, Chicago, IL \\ Joseph Krzak \\ Shriners Hospitals for Children, Chicago, IL \\ Lawrence Vogel \\ Shriners Hospitals for Children, Chicago, IL \\ Gerald F. Harris \\ Shriners Hospitals for Children, Chicago, IL
}

\begin{abstract}
Current methods for evaluating upper extremity (UE) dynamics during pediatric wheelchair use are limited. We propose a new model to characterize UE joint kinematics and kinetics during pediatric wheelchair mobility. The bilateral model is comprised of the thorax, clavicle, scapula, upper arm, forearm, and hand segments. The modeled joints include: sternoclavicular, acromioclavicular, glenohumeral, elbow and wrist. The model is complete and is currently undergoing pilot studies for
\end{abstract}


NOT THE PUBLISHED VERSION; this is the author's final, peer-reviewed manuscript. The published version may be accessed by following the link in the citation at the bottom of the page.

clinical application. Results may provide considerable quantitative insight into pediatric UE joint dynamics to improve wheelchair prescription, training and long term care of children with orthopaedic disabilities

\section{SECTION I.}

\section{Introduction}

Biomechanical analysis has been used by many to evaluate upper extremity (UE) motion during human movement, including during the use of assistive devices such as crutches and walkers. ${ }^{1,2}$ However, few studies have been conducted to examine the UE kinetics during wheelchair mobility.3,4

In $2000,90 \%$ of wheelchair users (1.5 million people) in the United States (US) were manual wheelchair users (MWU), requiring the use of their upper body to maneuver the wheelchair as well as to perform other activities of daily living. Among children under the age of 18 , the wheelchair was the most used assistive mobility device at $0.12 \%$ of the US population (about 88,000 children). Of these, 89.9\% $(79,000)$ used manual wheelchairs. ${ }^{5}$

Associated with leading causes of assistive device usage in children and adolescents, are severe cases of osteogenesis imperfecta (01), cerebral palsy (CP), myelomeningocele (MM) and spinal cord injury (SCI). Once confined to a wheelchair, the UEs must take over the responsibilities of locomotion as well as maintain the ability to perform activities of daily living. The UE bones and joints are not constructed for this load magnitude or frequency, so overuse injuries such as: carpal tunnel syndrome, shoulder impingement and UE pain are common.6,7 Due to increased life expectancy and continual wheelchair use, these injuries may reduce or severely limit independent function and quality of life. ${ }^{6}$

Better knowledge of UE dynamics during wheelchair propulsion may improve our understanding of the onset and propagation of UE pathologies. This may lead to improvements in wheelchair prescription, design, training, and long-term/transitional care. Thereby, pathology onset may be slowed or prevented, and quality of life restored.

The goals of this work are to create an UE biomechanical model, based on the work of Slavens et al. ${ }^{8}$ including: additional segments, more accurate representations of segments and joint locations, and kinetic modeling to determine UE joint forces and 
NOT THE PUBLISHED VERSION; this is the author's final, peer-reviewed manuscript. The published version may be accessed by following the link in the citation at the bottom of the page.

moments. The model is specifically designed for the pediatric MWU with existing and potential UE pathology.

\section{SECTION II.}

\section{Methods}

\section{A. Kinematic Model}

The bilateral UE model is comprised of 11 segments, including: thorax, clavicles, scapulae, upper arms, forearms and hands. The joints of interest are: three degree-offreedom wrist, glenohumeral, and acromioclavicular joints; and two-degree-of-freedom sternoclavicular and elbow joints. Twenty-seven passive reflective markers are placed on bony anatomical landmarks to reduce skin motion artifact while defining the aforementioned segments (Fig. 1).

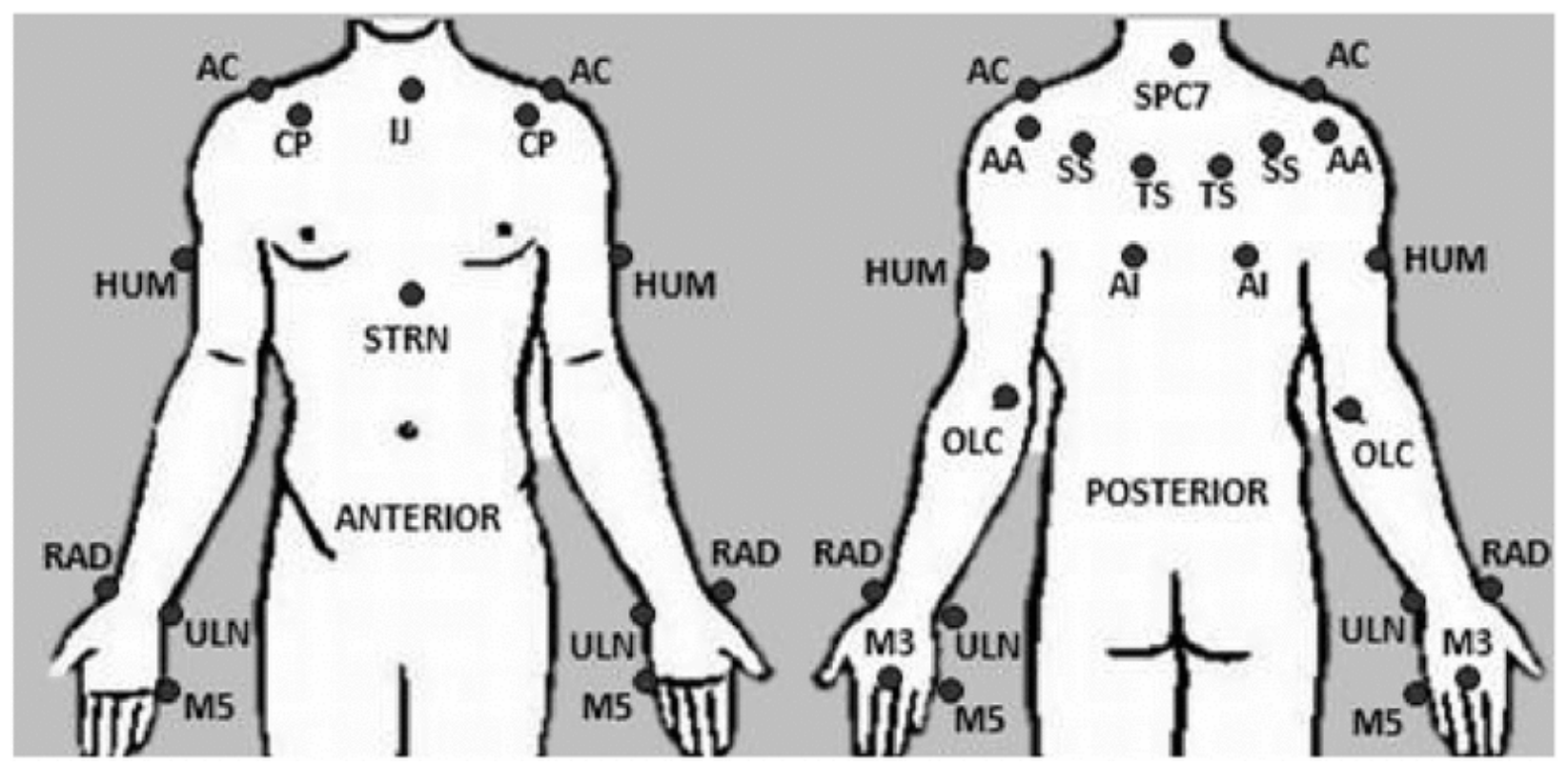

Figure 1. UE model marker set: IJ: suprasternal notch, STRN: xiphoid process, SPC7: spinal process C7, AC: acromioclavicular joint, AI: inferior angle, TS: trigonum spine, SS: scapular spine, AA: acromial angle, CP: coracoid process, HUM: humerus technical marker, OLC: olecranon, RAD: radial styloid, ULN: ulnar styloid, M3 and M5: third and fifth metacarpals.

The wheelchair is modeled as one rigid body segment using four markers. Joint axes are embedded at the joint centers which are calculated using subject specific anthropometric data. A Z-X-Y Euler sequence is used to determine the joint angles of the 
distal segment with respect to the proximal segment. Following ISB recommendations, the Z-axis points laterally towards the subject's right side, the X-axis points anteriorly and the Y-axis points superiorly. ${ }^{9}$ Vicon BodyBuilder (Vicon Motion Systems, Ltd., Oxford, England) and Matlab (MathWorks, Inc., Massachusetts, USA) were used for model development.

\section{B. Model Features}

A previous UE model for wheelchair evaluation created by our group ${ }^{8}$ served as the foundation for the development of this model. Several new design features were developed in order to better define shoulder complex kinematics, quantify UE joint kinetics and improve accuracy. First, in order to avoid possible marker contact with the wheelchair during propulsion, a single marker was placed on the olecranon, a method previously validated by Hingtgen et al. ${ }^{10}$ Next, the marker set used to describe the thorax was updated to more closely reflect the model described by Nguyen et al. in which the direct method of marker placement on thorax landmarks reduces the influence of shoulder girdle movement on thoracic kinematic measurements. ${ }^{11}$ In order to improve accuracy the method of determining the glenohumeral joint center location was modified from using the shoulder circumference to regression equations developed by Meskers et al. that employ the positions of five scapula markers. ${ }^{12}$

Additionally, with the inclusion of the scapula segments, a new marker tracking method for the TS and AI scapula markers is used to reduce the effects of skin motion artifact and possible marker-wheelchair interaction, using techniques as developed by Senk et al. ${ }^{13}$

Lastly, the body segment parameters were chosen to be calculated through equations developed specifically for the pediatric population.

\section{Joint Centers}

The positions of the joint centers were the origins for each segment's local coordinate system, except for the scapulae and forearms, whose origins are located at the AA and ULN markers respectively, following ISB recommendations. ${ }^{9}$ The thorax origin is located halfway between the IJ and SPC7 markers. All joints were assumed to have fixed centers of rotation. 


\section{Segment Coordinate Systems}

Segment coordinate systems (SCS) were determined for each of the model's 11 segments. The joint angles were determined by the relative motion between two adjacent SCS, distal relative to proximal. The SCS follow the right-hand rule with the Z-axis as the flexion/extension axis; the $\mathrm{X}$-axis as the abduction/adduction axis; and the $\mathrm{Y}$-axis as the internal/external rotation axis. Equations presented define the right side; the left side of the model was similarly defined. The symbol $\bar{m}_{\text {subscript }}$ represents the individual markers as defined in figure 1 .

$\operatorname{Thorax}(t)$

The following unit vectors defined the axes of the thorax segment. $t_{c}$ : thorax origin, $p_{t}$ : temporary point (the temporary point is located to the right of the thorax origin and was created in order to properly define the orientation of the thorax coordinate system).

$$
\begin{aligned}
& \bar{\tau}_{\chi}=\frac{\bar{m}_{I J}-\bar{m}_{S P C 7}}{\left\|\bar{m}_{I J}-\bar{m}_{S P C 7}\right\|} \\
& \bar{r}_{y}=\frac{\bar{p}_{t}-\bar{t}_{c}}{\left\|\bar{p}_{t}-\bar{t}_{c}\right\|} \times \bar{\tau}_{t, x}
\end{aligned}
$$

$(1)(2)$

\section{Clavicle (c)}

The following unit vectors defined the axes of the right clavicle segment.

$$
\begin{gathered}
\bar{C}_{z}=\frac{\bar{m}_{A C}-\bar{m}_{I J}}{\left\|\bar{m}_{A C}-\bar{m}_{I J}\right\|} \\
\bar{C}_{\chi}=\bar{r}_{y} \times \bar{C}_{z}
\end{gathered}
$$

(3)(4) 


\section{Scapula (s)}

The following unit vectors defined the axes of the right scapula segment.

$$
\begin{gathered}
\bar{s}_{Z}=\frac{\bar{m}_{A A}-\bar{m}_{T S}}{\left\|\bar{m}_{A A}-\bar{m}_{T S}\right\|} \\
\bar{s}_{x}=\frac{\bar{m}_{A A}-\bar{m}_{A I}}{\left\|\bar{m}_{A A}-\bar{m}_{A I}\right\|} \times \bar{s}_{Z}
\end{gathered}
$$

Upper Arm (ua)

The following unit vectors defined the axes of the right upper arm segment. $G H_{c}$ : glenohumeral joint center, $e_{c}$ : elbow joint center

$$
\begin{gathered}
\overline{u a}_{y}=\frac{\overline{G H}_{C}-\bar{e}_{c}}{\left\|\overline{G H}_{C}-\bar{e}_{c}\right\|} \\
\overline{u a}_{Z}=\frac{\bar{m}_{U L N}-\bar{e}_{c}}{\left\|\bar{m}_{U L N}-\bar{e}_{c}\right\|} \times \overline{u a}_{y}
\end{gathered}
$$

Forearm ( $f$ )

The following unit vectors defined the axes of the right forearm segment.

$$
\begin{gathered}
\overline{f_{y}}=\frac{\bar{e}_{c}-\bar{m}_{U L N}}{\left\|\bar{e}_{c}-\bar{m}_{U L N}\right\|} \\
\overline{f_{\chi}}=\frac{\bar{m}_{U L N}-\bar{m}_{R A D}}{\left\|\bar{m}_{U L N}-\bar{m}_{R A D}\right\|} \times \overline{f_{\mathrm{y}}}
\end{gathered}
$$

Hand (h)

The following unit vectors defined the axes of the right hand segment. $w_{c}$ : wrist joint center, 3c: third metacarpal joint center 
NOT THE PUBLISHED VERSION; this is the author's final, peer-reviewed manuscript. The published version may be accessed by following the link in the citation at the bottom of the page.

$$
\begin{gathered}
\bar{h}_{y}=\frac{\bar{w}_{c}-\bar{m}_{3 C}}{\left\|\bar{w}_{C}-\bar{m}_{3 C}\right\|} \\
\bar{h}_{X}=\frac{\bar{m}_{U L N}-\bar{m}_{R A D}}{\left\|\bar{m}_{U L N}-\bar{m}_{R A D}\right\|} \times \bar{h}_{y}
\end{gathered}
$$

\section{E. Kinetic Model - Body Segment Parameters}

To solve for the forces and moments occurring at the wrist, elbow and glenohumeral joints, the Newton-Euler equations of motion used for the inverse dynamics method require an array of inputs. The kinematic model provides the necessary information regarding segment and joint movement; however, reactionary forces and subject specific body segment parameters must also be determined. The body segment parameters required for each segment include: mass, center of mass location and inertia.

To determine the mass of the hands, forearms and upper arms, equations developed by Jensen et al. were used. ${ }^{14}$ These equations use the subject's age (in years) as the independent variable to determine the mass proportion of the segment to the body. The equations were determined for patients between the ages of four and twenty. Jensen et al. ${ }^{14}$ also developed polynomial regression equations based on age for the calculation of the location of the segment center of mass, which we applied.

Lastly, in order to determine the segment inertias for each individual subject, equations developed by Yeadon and Morlock et al. were utilized. ${ }^{15}$ These equations require many subject measurements for proper application.

\section{F. Kinetic Model - Newton - Euler Equations}

Commercialization of the SmartWheel, produced through Three Rivers Holdings LLC. (Mesa, AZ, USA), now enables kinetic data to be recorded during wheelchair propulsion. The Smart Wheel utilizes voltage changes in six strain gauges placed on specialized wheel spokes, called beams, to calculate the three forces and three moments as applied by the hand to the wheelchair handrim. This data may be used in the Newton-Euler equations of motion in order to determine the forces and moments at each UE joint of interest through the inverse dynamics method. ${ }^{16}$ 
Through the use of these equations, the forces and moments in all three planes of motion are calculated for each joint. Below are the calculations for the wrist joint; similar calculations were conducted for the elbow and glenohumeral joints.

\section{Hand}

The wrist force, $\bar{F}_{W}$, is computed by summing the force due to linear acceleration of the hand center of mass, the force of the hand due to gravity and the resultant handrim reaction force, $F_{S W}$, determined by the SmartWheel:

$$
\bar{F}_{W}=-\operatorname{mass}_{H}\left(\bar{a}_{H}+\bar{g}\right)-\bar{F}_{S W}
$$

where mass $H$ is the mass of the hand, $\bar{a}_{H}$ is the acceleration vector of the hand center of mass and $\bar{g}$ is acceleration vector due to gravity $\left(-9.81 \mathrm{~m} / \mathrm{s}^{2}\right)$.

The wrist moment, $\bar{M}_{W}$, is computed by adding the moment occurring about the hand center of mass due to the resultant handrim reaction force, $M_{D i s H}$ and subtracting the moment occurring about the hand center of mass due to the wrist force, $M_{\text {ProxH }}$ and the known reactionary moment provided by the SmartWheel, $M_{S W}$ to the rate of change of angular momentum of the hand, $H_{H}$ as determined by the subject specific equations.

$$
\bar{M}_{W}=H_{H}-\bar{M}_{S W}+\bar{M}_{D i s H}-\bar{M}_{\text {ProxH }}
$$

where $M_{\text {DisH }}$ and $M_{\text {ProxH }}$ are defined below, with $r_{\text {ProxH }}$ and $r_{\text {DisH }}$ representing the moment arms from hand center of mass to the wrist joint and metacarpal joint respectively.

$$
\begin{aligned}
\bar{M}_{\text {ProxH }} & =\bar{r}_{\text {ProxH }} \times \bar{F}_{W} \\
\bar{M}_{\text {DisH }} & =\bar{r}_{\text {DisH }} \times \bar{F}_{S W}
\end{aligned}
$$

Similar equations are solved for the remainder of the joints.

SECTION III.

\section{Model results and Refinements}

\section{A. Protocol}

2012 Annual International Conference of the IEEE Engineering in Medicine and Biology Society (EMBC), (2012): pg. 4788-4791. DOI. This article is (C) Institute of Electrical and Electronics Engineers (IEEE) and permission has been granted for this version to appear in ePublications@Marquette. Institute of Electrical and Electronics Engineers (IEEE) does not grant permission for this article to be further copied/distributed or hosted elsewhere without the express permission from Institute of Electrical and Electronics Engineers (IEEE). 
Previous research by our group investigated bilateral kinematics of children with spinal cord injury (SCI) during wheelchair mobility. ${ }^{8}$ Thirteen manual wheelchair users (MWU) with SCI, aged 9-25 years of age, participated in the study. Each subject propelled their wheelchair along a 15 meter walkway at a self-selected speed for multiple trials, with adequate rest provided between trials. Motion data was collected at $120 \mathrm{~Hz}$ using a 14 camera Vicon MX motion capture system.

\section{B. Results}

Group mean joint angles of the glenohumeral, elbow and wrist joints were characterized over the wheelchair stroke cycle, with $100 \%$ stroke cycle defined by push and recovery phases. Mean glenohumeral joint angles in each plane of motion, along with + / - one standard deviation are depicted in Fig. 2.8 The mean peak angles and ranges of motion (ROMs) of each joint were also computed over the stroke cycle. Two sample t-tests were conducted to assess asymmetry. ${ }^{8}$

2012 Annual International Conference of the IEEE Engineering in Medicine and Biology Society (EMBC), (2012): pg. 4788-4791. DOI. This article is (C) Institute of Electrical and Electronics Engineers (IEEE) and permission has been granted for this version to appear in ePublications@Marquette. Institute of Electrical and Electronics Engineers (IEEE) does not grant permission for this article to be further copied/distributed or hosted elsewhere without the express permission from Institute of Electrical and Electronics Engineers (IEEE). 
(a)

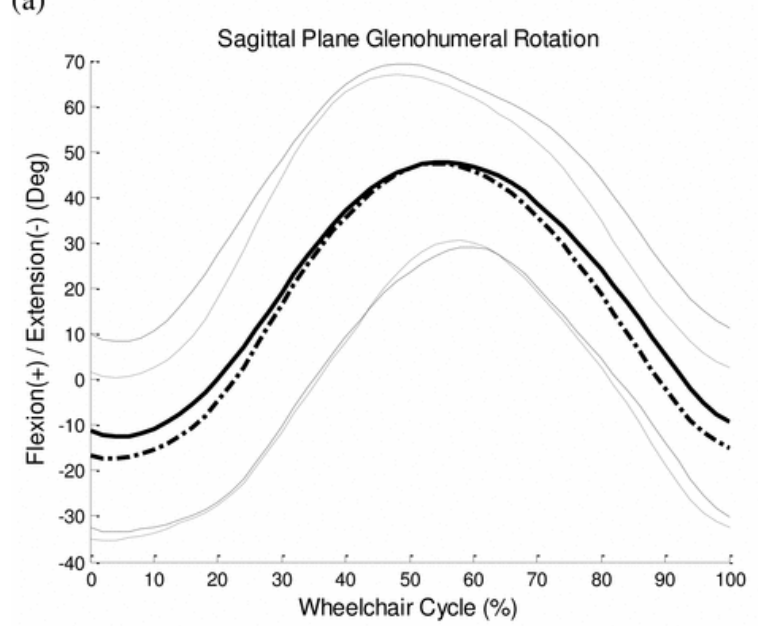

(b)

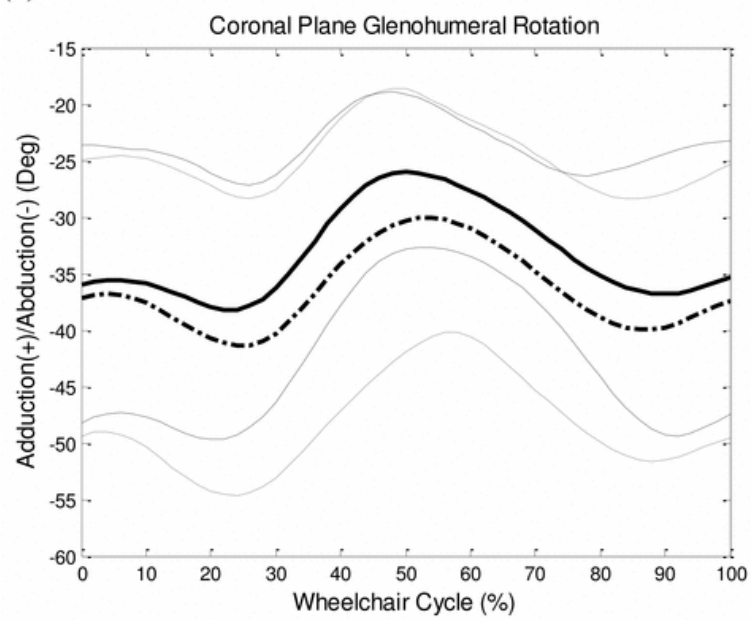

Figure 2. Mean (bold) and +/- 1 SD of bilateral glenohumeral joint kinematics in the (a) sagittal plane, (b) coronal plane and (c) transverse plane. Left is solid and right is dashed.

Findings from the kinematic studies are incorporated into the refined model which provides complete DE joint kinetics. The refinements also include additional kinematic data for the acromioclavicular and sternoclavicular joints

\section{Discussion}

Current findings from our modeling demonstrate joint ranges of motion to range from $13^{\circ}$ at the wrist in the transverse plane to $72^{\circ}$ at the shoulder in the sagittal plane. The large joint ROMs seen at the shoulder, as well as the elbow, highlight a heightened concern about increased joint demands during manual wheelchair propulsion in the developing joints of pediatric users. Since inappropriate UE positioning in conjunction with increased joint load demands may lead to early onset pain and pathology in children, the 
refined model was constructed to reveal detailed joint kinetic demands while also incorporating a more sophisticated and biofidelic representation of anatomic shoulder articulations.

Results from the preliminary development and patient assessment studies indicated a need for additional, detailed asymmetry assessment throughout the propulsive cycle with information on internal joint demands (forces and moments). Kinetic quantification approximately doubles the amount of quantitative information available for assessment of UE joint asymmetry effects, such as those resulting from limb dominance, anthropometry, pathology, antalgia and maneuvering demands.

It is expected that the proposed model will improve our understanding of complex shoulder dynamics due to the addition of anatomically relevant shoulder complex segments and more accurate subject specific segment parameter determination. Additionally, new methods for joint center locations are employed to further increase angular (and thus kinetic) data accuracy.

Comparisons to current reports of adult pathological populations ${ }^{3,4}$ may provide new insight into UE joint demands within the pediatric population of MWU. The kinetic data may additionally offer new insight into the specific dynamic joint demands for wheelchair propulsion and maneuverability. This in turn will be of great value as we investigate the correlation of joint demands to overuse injuries within the pediatric population. This work may ultimately lead to a reduction in UE pain and pathology in pediatric MWUs.

\section{Acknowledgment}

We acknowledge OREC and Shriners Hospitals for Children, Chicago, for their support. The contents of this work were developed under a grant from the Department of Education, NIDRR grant number H133E100007. However, the contents of this work do not necessarily represent the policy of the Department of Education, and you should not assume endorsement by the Federal Government.

\section{References}

1B.A. Slavens, et al., "Upper extremity dynamics during Lofstrand crutch-assisted gait in children with myelomeningocele," Gait And Posture, vol. 30, pp. 511-17, 2009.

${ }^{2}$ K.A. Konop, et al., "A biomechanical analysis of upper extremity kinetics in children with cerebral palsy using anterior and posterior walkers," Gait And Posture, vol. 30, no. 3, pp. 265-394, 2009. 
3J. L. Mercer, M. Boninger, et al., "Shoulder joint kinetics and pathology in manual wheelchair users.," Clinical Biomechanics (Bristol, Avon), vol. 21, no. 8, pp. 781-9, Oct. 2006.

${ }^{4}$ A. Gil-Agudo, et al., "Upper limb joint kinetics during manual wheelchair propulsion in patients with different levels of spinal cord injury.," Journal Of Biomechanics, vol. 43, no. 13, pp. 2508-15, Sep. 2010.

${ }^{5}$ H. S. Kaye, et al. "Mobility Device Use in the United States," Disability Statistics Report, Washington, DC: US Dept Education, NIDRR., vol. 14. pp. 1-60, 2000.

${ }^{6}$ B. Crane, In M.M. Lusardi, and C. C. Nielsen, Orthotics And Prosthetics In Rehabilitation St. Louis, MS: Saunders Elsevier. Ed. 2, pp. 489-516, 2007.

${ }^{7}$ H. E. J. Veeger, et al., "Wrist motion in handrim wheelchair propulsion.," Journal Of Rehabilitation Research And Development, vol. 35, no. 3, pp. 305-13, Jul. 1998.

8B. Slavens, et al., "Upper extremity wheelchair kinematics in children with spinal cord injury," Proc. Ieee Embs, Boston, Ma, 2011.

${ }^{9} \mathrm{G}$. Wu, et al., "ISB recommendation on definitions of joint coordinate systems of various joints for the reporting of human joint motion - Part II: shoulder, elbow, wrist and hand," Journal of Biomechanics, vol. 38, pp. 981-92, 2005

${ }^{10}$ B. Hingtgen, et al., "An upper extremity kinematic model for evaluation of hemiparetic stroke.," Journal Of Biomechanics, vol. 39, no. 4, pp. 681-8, Jan. 2006.

11T. C. Nguyen and R. Baker, "Two methods of calculating thorax kinematics in children with myelomeningocele.," Clinical Biomechanics (Bristol, Avon), vol. 19, no. 10, pp. 1060-5, Dec. 2004.

${ }^{12} \mathrm{C}$. G. Meskers, et al., "In vivo estimation of the glenohumeral joint rotation center from scapular bony landmarks by linear regression.," Journal Of Biomechanics, vol. 31, no. 1, pp. 93-6, Jan. 1998.

${ }^{13} \mathrm{M}$. Senk and L. Chèze, "A new method for motion capture of the scapula using an optoelectronic tracking device: a feasibility study.," Computer Methods In Biomechanics And Biomedical Engineering, vol. 13, no. 3, pp. 397-401, Jun. 2010.

${ }^{14}$ R. K. Jensen, "Changes in segment inertia proportions between 4 \& 20 years," Journal Of Biomechanics, vol. 22, no. 6/7, pp. 529-36, 1989.

${ }^{15}$ M. R. Yeadon and M. Morlock, "The appropriate use of regression equations for the estimation of segmental inertia parameters", Journal Of Biomechanics, vol. 22, no. 6/7, pp. 683-89, 1989.

${ }^{16}$ V. M. Zatsiorsky, Kinetics Of Human Motion. Champaign, IL: Human Kinetics. 2002. 